\begin{tabular}{lc} 
Sharif University of Technology & Scientia Iranica \\
SCIENTIA & Transactions F: Nanotechnology \\
I RAN ICA & http://scientiairanica.sharif.edu \\
\hline
\end{tabular}

\title{
Fusarium oxysporum, a bio-factory for nano selenium compounds: Synthesis and characterization
}

\author{
F. Asghari-Paskiabi ${ }^{a}$, M. Imani ${ }^{\mathrm{b}, *}$, M. Razzaghi-Abyaneh ${ }^{\mathrm{c}, *}$, and H. Rafii-Tabar ${ }^{\mathrm{a}}$ \\ a. Department of Medical Physics and Biomedical Engineering, School of Medicine, Shahid Beheshti University of Medical Sciences, \\ Tehran, Iran. \\ b. Novel Drug Delivery Systems Department, Iran Polymer and Petrochemical Institute, Tehran, P.O. Box 14965/115, Iran. \\ c. Department of Mycology, Pasteur Institute of Iran, Tehran, 13164, Iran.
}

Received 2 October 2017; received in revised form 25 December 2017; accepted 20 January 2018

\section{KEYWORDS}

Fusarium oxysporum;

Selenium;

Nanoparticles;

Green synthesis;

Sulfite reductase;

Aerobic fermentation.

\begin{abstract}
Selenium nanoparticles have received major consideration for their antimicrobial and anticancer properties. In the present study, a mycelia fungus named Fusarium oxysporum was employed as a cell factory for bio-production of selenium and selenium sulfide nanoparticles. Scanning Electron Microscopy (SEM) micrographs displayed that the NPs were encompassed in medium and cells debris along with the presence of sulfur and selenium in the particles according to the energy-dispersive X-ray spectroscopy findings. The size of spherical NPs was measured between 34.32 and $231.98 \mathrm{~nm}$ in SEM micrographs and $81.9 \mathrm{~nm}$ in DLS analysis. Fourier-transform infrared spectroscopy spectra supported selenium compounds production and showed that proteins were associated with the particles. The presence of primary and secondary amine bands was demonstrated by the peaks at 1090-1020 and $1650-1580 \mathrm{~cm}^{-1}$, and at $1580-1490 \mathrm{~cm}^{-1}$ in FTIR spectra. UV/VIS spectrophotometry analysis showed that maximum absorbance for the test was at $217 \mathrm{~nm}$. The strongest Bragg's reflection in the X-ray diffractograms peaks revealed the closest match with $\mathrm{SeS}, \mathrm{SeS}_{2}$, and Se according to standard JCPDS cards. Taken together, our results show that, Fusarium oxysporum is able to produce selenium-based nanoparticles in a safe and cost effective aerobic green approach.

(C) 2018 Sharif University of Technology. All rights reserved.
\end{abstract}

\section{Introduction}

There are at least 25 human selenoproteins and enzymes which contain selenocysteine [1]. These proteins are necessary for avoiding cells damages and proper functions of the thyroid glands and immune system [2]. In comparison with selenium compounds, Se nanoparticles (NPs) have low cytotoxicity and show

\footnotetext{
*. Corresponding authors. Tel.: +9821 64112804, Fax: +98 2166465132

E-mail addresses: mrab442@pasteur.ac.ir ( $M$.

Razzaghi-Abyaneh); M.Imani@ippi.ac.ir (M.Imani)
}

marvelous anticancer and antimicrobial activities $[1,2]$. Also, because of semiconducting, photoelectrical, and catalytic properties of selenium NPs, their importance is increasing [1].

Application of selenium sulfide in treatment of skin diseases has an eighty-year history. Sulfur has fungicidal and therapeutic features itself and selenium is an activator that can increase the therapeutic activity of the former [3]. Selenium sulfide is basically used for treatment of dermatitis, folliculitis, and pityriasis, which are fungal infections [4]. Actually, until now, $\mathrm{S}^{0}$ has been the oldest known fungicide [5]. Enzymatic catalysis and radical scavenging of oxygen metabolites are often considered as the role of sulfur and selenium 
compounds in treatment of diseases such as pulmonary tuberculosis [6].

The conventional way of selenium sulfide production is chemical synthesis by fusing pure elements of sulfur and selenium at $350^{\circ} \mathrm{C}$ inside a vacuum Pyrex tube [7] or by heating of sulfur and selenium at $280^{\circ} \mathrm{C}$ for $2 \mathrm{~h}$ and cooling at $18-20^{\circ} \mathrm{C}$ [8]. Recently, a growing interest in Nano Particle (NP) biogenesis has appeared as a green technology to decrease the high cost and harmful sides of conventional chemical synthesis. To fulfill this goal, a lot of bio-systems such as plants, bacteria, fungi, and yeasts have been tried [9]. These microorganisms are able to recycle minerals in the earth and may tolerate and grow in high concentration of the metal salts. In NP formation process, the change of redox state leads to reduction in or removal of metal ions toxicity [10].

On the one hand, biological approaches have been used to synthesize selenium NPs as new, eco-friendly, and non-toxic approaches [2,11-13]. Fungi have also been employed to synthesize selenium NPs $[14,15]$ extracellularly. On other hand, Elemental sulfur is a widespread element, which is utilized by bacteria in several bio-reactions. For example, they use $\mathrm{S}_{8}$ as a source of energy by reducing it to sulfide. But, there is very low information of this phenomenon in eukaryotes [16]. Ahmad et al. [17] produced CdS NPs through extracellular enzyme of Fusarium oxysporum. They discovered that some eukaryotes such as fungi were able to synthesize CdS and other sulfide NPs by an enzymatic process. When $F$. oxysporum encountered $\mathrm{Cd}^{2+}$ and $\mathrm{SO}_{4}^{2-}$ in the medium, very stable CdS NPs were performed [17]. Abe et al. [16] discovered that $F$. oxysporum could reduce $\mathrm{S}_{8}$ anaerobically. They indicated that in lack of $\mathrm{O}_{2}$, dissimilation of $\mathrm{S}_{8}$ was very important for the fungus. They found, for the first time, that fungi had the ability of $\mathrm{S}_{8}$ reduction [16].

In this research, we examined the possibility of selenium compounds NPs synthesis by $F$. oxysporum in aerobic conditions. The obtained NPs were examined by $\mathrm{UV} /$ vis spectrophotometry, electron microscopy, Energy-Dispersive X-ray Spectroscopy (EDX), X-Ray Diffraction (XRD), Fourier-Transform Infra Red spectroscopy (FTIR), and Dynamic Light Scattering (DLS) techniques. We noticed that the produced NPs were made of Se and S.

\section{Experimental}

\subsection{Fungal strain and chemicals}

Fusarium oxysporum PFCC 115 was obtained from Mycology Department of Pasteur Institute of Iran. Glucose and malt extract were purchased from Merck, Germany. Sabouraud Dextrose Agar (SDA), peptone, and yeast extract were obtained from Micro Media, Hungary. Potato Dextrose Agar (PDA) was purchased from Scharlau Microbiology, Spain. Selenous acid was prepared by Sigma-Aldrich.

\subsection{Preparation and nanoparticle synthesis}

Fusarium oxysporum was cultured on PDA slant of which the spores were harvested and collected in tubes containing tween 80 in distilled water $(0.05 \%$ $\mathrm{V} / \mathrm{V})$. The spores were counted using a Neubauer Slide (HBG, Germany) under $40 \times$ objective lens of a light microscope. The spores were dispersed into the aqueous medium of malt extract-glucose-yeast extractpeptone [18] (MGYP; malt extract $0.3 \%$, glucose $1 \%$, yeast extract $0.3 \%$, and peptone $0.5 \%$ ) with the density of $10^{6}$ spores.mL ${ }^{-1}$. Incubation was performed at $30^{\circ} \mathrm{C}$ in $180 \mathrm{rpm}$ using a shaking incubator (LabTech DAIHAN LABTECH CO., LTD, LSI-3016 R, Korea). On the 5th day, the fungal elements were separated with 2 layers of sterile gauzes. First, the fungal mat was washed with sterile distilled water several times. Then, the mat of the test was transferred to a sterile (filtered with 0.22-micron disk filters, JET BIOFIL $^{\circledR}$, Canada) solution of selenous acid (3 mM) and incubated at $30^{\circ} \mathrm{C}$, shaking in $180 \mathrm{rpm}$. The biomass of control was behaved like the test, except that it was transferred to sterile distilled water. After 48 hours and observing the color change, the content of the Erlenmeyer was centrifuged (3000 rpm, $6 \mathrm{~min}$, $15^{\circ} \mathrm{C}$ ) and the precipitates were collected and dried at $70^{\circ} \mathrm{C}$ overnight and then, the characterization analyses were performed on them.

\subsection{Characterization of nanoparticles}

Scanning Electron Microscopy (SEM) (MIRA3 TESCAN) analysis was performed on NP suspensions, which were dried and gold sputtered on a glass or carbon substrate. Observations were carried out at an accelerating voltage of $50.0 \mathrm{kV}$.

A Philips Analytical X'Pert MPD instrument operated at a voltage of $40 \mathrm{kV}$ and a current of 40 $\mathrm{mA}$ with $\mathrm{Cu} \mathrm{K}-\alpha(1.54 \AA)$ radiation was used for XRD measurements. For FTIR analysis, the potassium bromide pellet technique was used for preparation. For measurements, a Nicolet IR 100 instrument was employed at a spectral resolution of $1 \mathrm{~cm}^{-1}$ in a wavelength range of 1800 to $600 \mathrm{~cm}^{-1}$. DLS (Malvern Instruments Ltd, ZEN 3600) analysis was performed on NPs suspensions with an OD of approximately 0.3 at the wavelength of $630 \mathrm{~nm}$ after 10-min sonication in bath sonicator (DAIHAN LABTECH CO., LTD., LUC-410, Korea). To record the absorption spectra of the NPs suspension, a spectrophotometer, Shimadzu, UV-1601PS, Japan, was utilized. The wavelength scanning was performed from 100 to $1100 \mathrm{~nm}$ for the initial test and the test after diluting in ratios of $1: 2$, $1: 3,1: 6$, and $1: 12$ in distilled water $(\mathrm{v} / \mathrm{v})$ to obtain the 
maximum absorption under 1. Distilled water was used as a blank.

\section{Results and discussion}

The obtained NPs were characterized by UV/Vis spectrophotometry, SEM, EDX, XRD, FTIR, and DLS. Color change of the Erlenmeyer of the test was observed $48 \mathrm{~h}$ after adding 5 -day fungal elements into the $3 \mathrm{mM}$ selenous acid solution and incubation at $30^{\circ} \mathrm{C}$ with shaking in $180 \mathrm{rpm}$ (Figure 1). UV/VIS spectrophotometry analysis showed that maximum absorbance for test was at $217 \mathrm{~nm}$ (Figure 2). Sulfide and thiols maximum absorption was in the range of 200 to $220 \mathrm{~nm}$ [19], which could approve the presence of selenium sulfide. Ahmad et al. [17] observed the color change arising from CdS NPs formation 6 days after $\mathrm{Cd}^{2+}$ and $\mathrm{SO}_{4}^{2-}$ encountering. They attributed the spectrophotometry Optical Density (OD) at 450

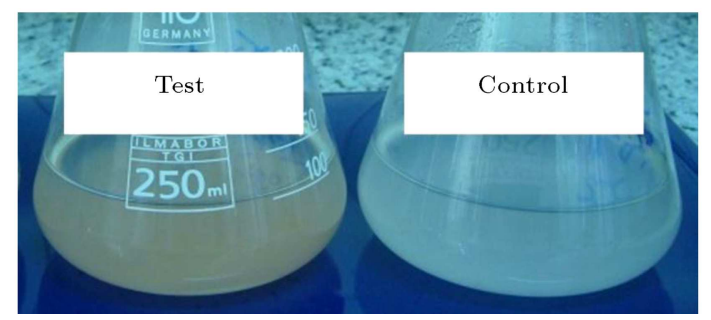

Figure 1. Macroscopic proof of selenium compounds NPs biosynthesis. The biomass of $F$. oxysporum encountered $3 \mathrm{mM}$ selenous acid. Color change happened after 48 hours (Test). To determine the difference, a negative control was prepared in which no salt had been added (Control).

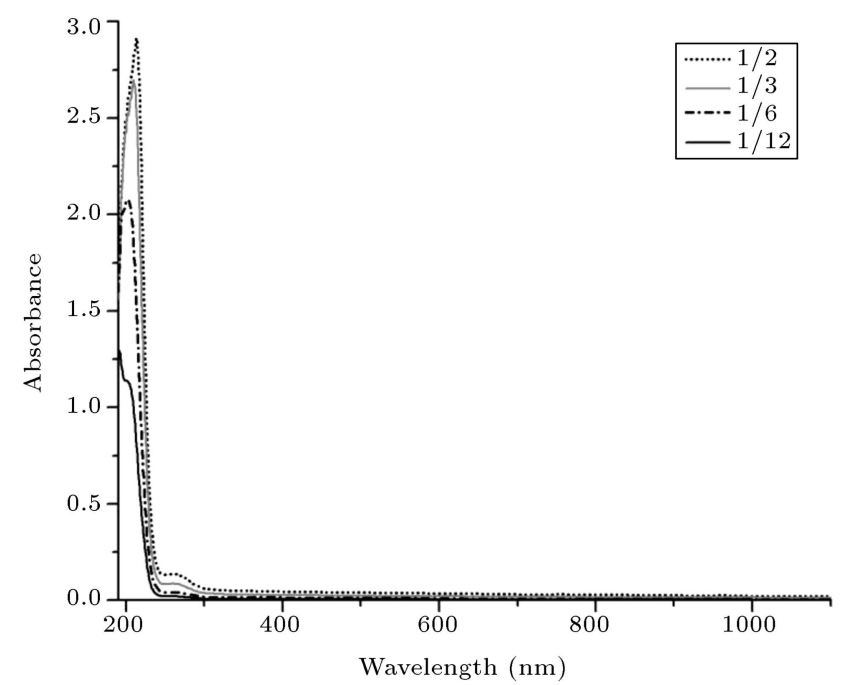

Figure 2. Wavelength scanning of biosynthesized selenium compounds NPs. Wavelength scanning of selenium compounds NPs was performed after diluting them with the ratios of 1:2 (purple), 1:3 (red), 1:6 (black), and 1:12 (blue) in distilled water (V/V). The OD of initial test was more than 1 , which is not shown here. $\mathrm{nm}$ to quantum size of CdS particles and an OD at $280 \mathrm{~nm}$ to the presence of proteins in the medium. The proteins caused a long-lasting stability of $\mathrm{CdS}$ NPs, because they attached to the NPs surfaces and prevented their accumulation [17]. Reyes et al. [20] also produced CdS NPs using Fusarium sp. taken from an ecosystem containing Cd (II). They observed OD at 450 and $264 \mathrm{~nm}$ and attributed them to CdS and NPs, respectively. Particles with smaller sizes had OD in smaller wavelengths, which showed the quantum size effects of the particles. The size of NPs was between 80 and $120 \mathrm{~nm}$. Some particles with sizes of 350 to 390 $\mathrm{nm}$ were observed, too; the authors believed that they were because of thiol groups of the cell walls [20].

The XRD pattern of the NPs taken after $48 \mathrm{~h}$ reactions is presented in Figure 3. The peaks marked with an asterisk had the strongest Bragg's reflection, which revealed the closest match with $\mathrm{SeS}, \mathrm{SeS}_{2}$, and Se according to standard JCPDS cards 00-002-0320, 00-047-1481, and 00-042-1425, respectively. Addition of $\mathrm{S}$ to Se led to a drop in Se crystallization [7]. The presence of 3-5 at.\% sulfur in selenium structure could reduce the rate of selenium crystallization [8]. In fact, the crystallinity of selenium-sulfur was dominated by sulfur proportion in the conformation. For example, small amounts of sulfur up to $28 \%$ deviated from the diffraction pattern, while increasing it up to $50 \%$ completely changed the profile of the diffraction pattern. Also, the increased number of peaks represented addition of some phases attributed to selenium sulfide. Because of selenium's five potential oxidation states $(-2,0,+2,+4$, and +6$)$, it can be introduced in several compounds with different chemical properties.

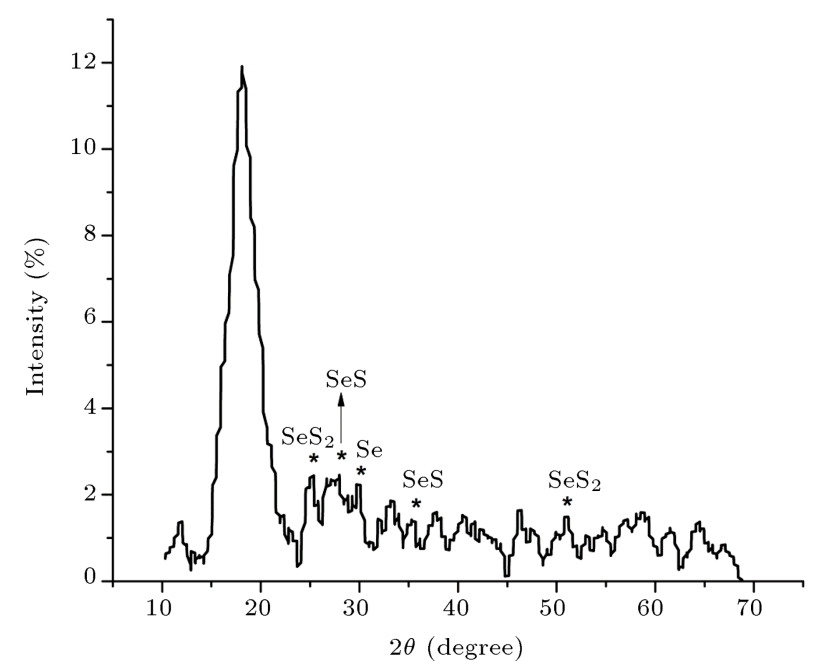

Figure 3. XRD analysis of biosynthesized selenium compounds NPs. The X-ray diffractogram was taken from the precipitates of NPs and $F$. oxysporum biomass after $48 \mathrm{~h}$ reaction, which were collected and dried at $70^{\circ} \mathrm{C}$ overnight. The peaks marked with an asterisk disclosed the match with $\mathrm{SeS}, \mathrm{SeS}_{2}$, and Se. 


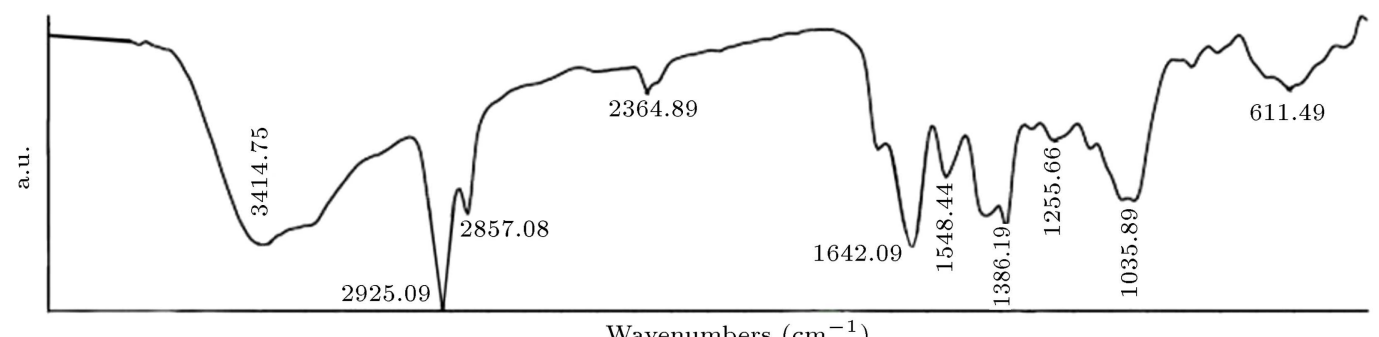

Wavenumbers $\left(\mathrm{cm}^{-1}\right)$

Figure 4. FTIR analysis of biosynthesized selenium compounds NPs. The FTIR analysis was performed on the precipitates of NPs and F. oxysporum biomass after $48 \mathrm{~h}$ reaction, which were collected and dried at $70^{\circ} \mathrm{C}$ overnight. The peaks at $1090-1020$ and $1650-1580 \mathrm{~cm}^{-1}$, and at $1580-1490 \mathrm{~cm}^{-1}$ showed the presence of primary and secondary amine bands [22]. Peaks around 600 to 650 and 1550 are attributed to sulfide compounds [18,19].

Moreover, in approximately all organic and inorganic sulfur compounds, selenium can substitute sulfur [21].

The peaks at $1090-1020$ and $1650-1580 \mathrm{~cm}^{-1}$, and at $1580-1490 \mathrm{~cm}^{-1}$ in FTIR spectra demonstrated the presence of primary and secondary amine bands [22] of proteins, which encompassed the NPs (Figure 4).

Rajput et al. (2016) observed the very stable NPs within weeks after reaction of silver nitrate and F. oxysporum. They demonstrated that bio-silver NPs were more stable than the commercial ones. Also, the bio-NPs had a 3-times larger hydrodynamic diameter because of capping layers of biomolecules, which were produced by $F$. oxysporum. FTIR and/or SDS/PAGE analysis showed that the proteins were responsible for this stability. In fact, the high surface energy of NPs results in adsorption of biomolecules from biological fluids. These biomolecules may be displaced by the new ones over time [23]. Almeida et al. (2017) also demonstrated the presence of amide I and II and aromatic groups in the filtrate of $F$. oxysporum, which they had applied to bio-synthesize silver NPs [24]. Yamaguchi et al. (2016) observed that after production of biogenic CdSe quantum dots by $F$. oxysporum, in the superoxide evaluation, superoxide dismutase (SOD) in the cytoplasm decreased. They attributed these changes to the creation of CdSe quantum dots in $F$. oxysporum [25]. Peaks around 600 to 650 and at 1550 in FTIR could be attributed to sulfide compounds according to $[18,19]$, while EDX analysis of the NPs approved the existence of selenium and sulfur spectral peaks (Figure 5).

The size of spherical NPs surrounded by fungal elements in SEM micrographs was measured between 34.32 and $231.98 \mathrm{~nm}$ (Figure 6), where the average hydrodynamic diameter of the NPs obtained from DLS analysis was $81.9 \mathrm{~nm}$.

Generally, sulfur assimilation is a path for the synthesis of sulfur-containing defense compounds (SDCs). SDCs are vital for saving the plants against various stresses. Some SDCs are elemental sulfur $\left(\mathrm{S}^{0}\right), \mathrm{H}_{2} \mathrm{~S}$, glutathione, phytochelatins, and sulfur-rich proteins. Their formation is closely dependent on sulfate uptake and assimilation [5]. Fungi first uptake the sulfate

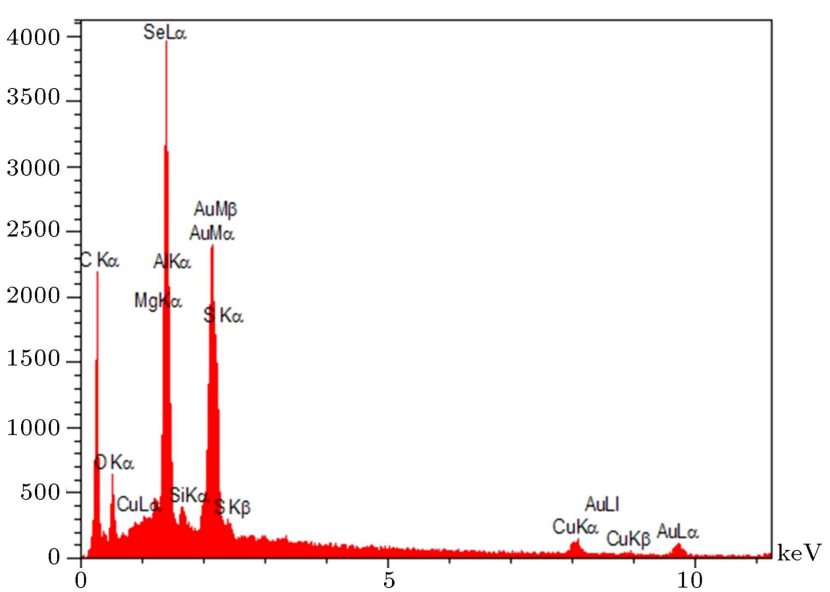

Figure 5. EDX analysis of biosynthesized selenium compounds NPs. The EDX analysis was performed on the precipitates of NPs and F. oxysporum biomass after $48 \mathrm{~h}$ reaction, which were collected and dried at $70^{\circ} \mathrm{C}$ overnight. SEM-EDX of NPs exposed the presence of spectral peak for selenium and sulfur in the test.

from the environment and then transport it inside by sulfate transporters. The activation happens by ATP of ATP sulfurylase and the product is APS (adenylyl sulfate). APS reductase reduces the APS in which a tripeptide glutathione (GSH) acts as an electron donor. Then, an enzyme, namely, sulfite reductase, reduces the produced sulfite to $\mathrm{H}_{2} \mathrm{~S}[5,26]$. We supposed that when the microorganism encountered a foreign compound, the aforementioned process happened and the fungus absorbed the compound and produced a metal/element sulfide compound instead of $\mathrm{H}_{2} \mathrm{~S}$. In fact, this was a defensive reaction of the microorganism to survive. However, the sulfur source in the medium of our test was quite limited. Fungal cells respond to the deficiency of a nutrient, such as sulfur, in order to diminish its consequences. For example, in similar conditions, in Neurospora crassa, which is a filamentous fungus, sulfur-related genes are expressed sequentially. While, when the sulfur of the medium is high, the entire set of genes is repressed. The main application of sulfate is its reduction to sulfide and biosynthesis of cysteine and methionine, subsequently. Arylsulfatase 


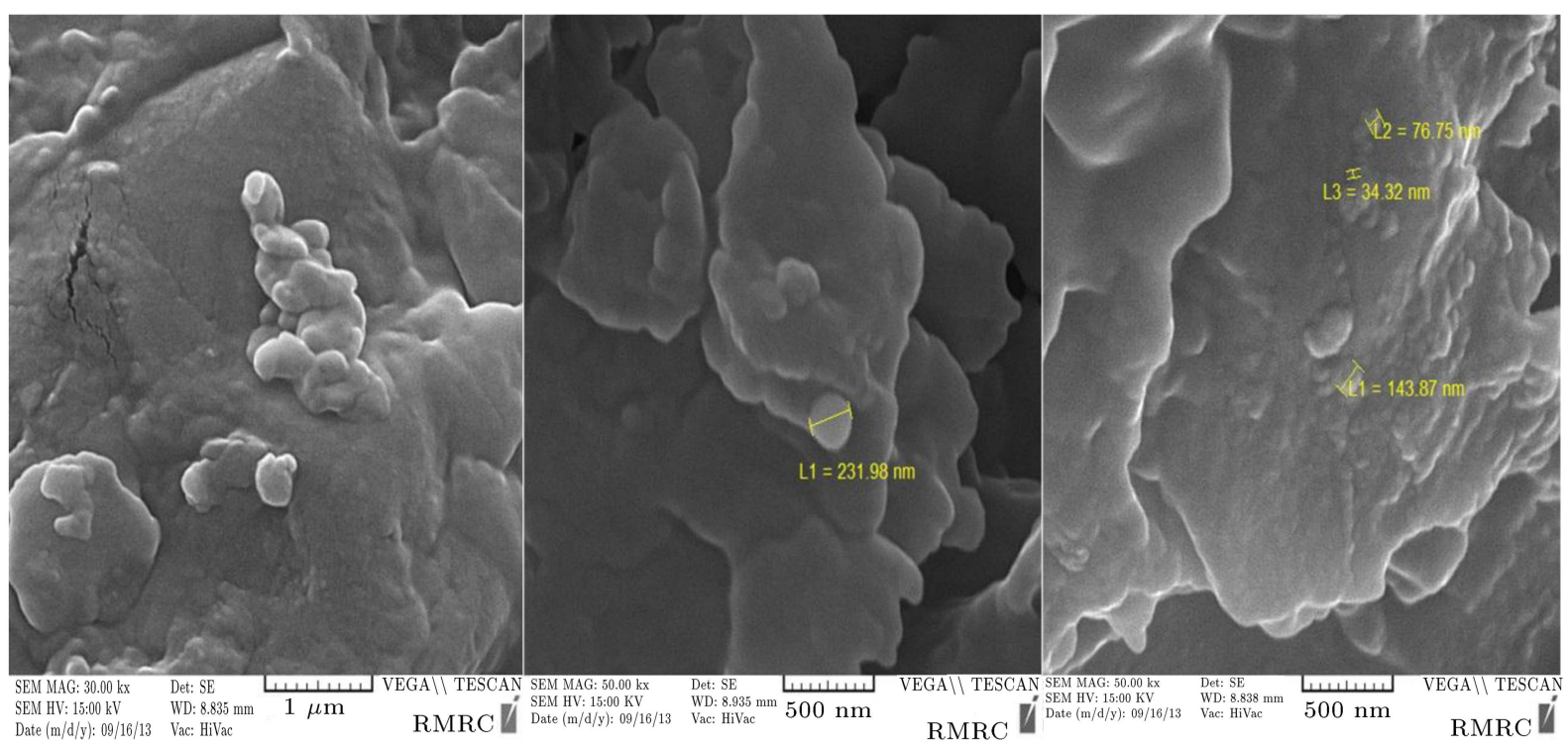

Figure 6. SEM micrographs of biosynthesized selenium compounds NPs. The SEM micrographs were taken from the precipitates of NPs and F. oxysporum biomass after $48 \mathrm{~h}$ reaction, which were collected and dried at $70^{\circ} \mathrm{C}$ overnight. The spherical NPs were encompassed inside the fungi debris.

enzyme activity of $N$. crassa was monitored for 14 hours in high- and low-sulfur media. Sulfur limitation resulted in a typical response to growth. After 6 hours, the enzyme activity increased by 5 folds, while in the high-sulfur condition, the enzyme activity had no fluctuation. It is supposed that a sensor protein is responsible for sulfur status detection in fungi. The sensor transmits a signal to a regulatory protein, which in turn regulates the genes involved in sulfur related responses. It is supposed that the metabolites of cysteine or methionine are the effectors, which are monitored by sulfur control system [27].

\section{Conclusions}

Taken together, main findings of the present study are as follows:

- Fusarium oxysporum, as a eukaryote, is able to produce selenium and selenium sulfide NPs;

- Selenous acid can be a precursor to biosynthesis of selenium-compounds NPs;

- $\mathrm{H}_{2} \mathrm{~S}$ production can be a defensive mechanism of the microorganism in confronting a foreign substance;

- In sulfur deficiency, the microorganism turns on all the genes needed to compensate for the loss;

- When Fusarium oxysporum confronts selenous acid as a foreign substance, it begins to reduce it, on the one hand, and produce sulfide-NP, on the other hand, to overcome the stress, while the sulfur related gens are expressed more in response to its deficiency.

\section{Acknowledgment}

This work was financially supported by a $\mathrm{PhD}$ fund to FAP from Shahid Beheshti University of Medical Sciences and a research grant (No. 766) to MRA from Pasteur Institute of Iran.

\section{References}

1. Wadhwani, S.A., Shedbalkar, U.U., Singh. R., and Chopad, B.A. "Biogenic selenium nanoparticles: current status and future prospects", Appl. Microbiol. Biotechnol., 100(1), pp. 2556-2566 (2016).

2. Sharma, G., Sharma, A.R., Bhavesh, R., Park, J., Ganbold. B., Nam, J.-S. and Lee S.-S. "Biomoleculemediated synthesis of selenium nanoparticles using dried Vitis vinifera (raisin) extract", Molecules, 19(3), pp. 2761-2770 (2014).

3. Nordlander, B.W, Chemical Products, US1860336 A: Google Patents (1932).

4. Mitchell, S.C., Nickson, R.M., and Waring, R.H. "The biological activity of selenium sulfide", Sulfur Reports, 13(2), pp. 279-289 (1993).

5. Rausch, T. and Wachter, A. "Sulfur metabolism: a versatile platform for launching defense operations", Trends Plant Sci., 10(10), pp. 503-509 (2005).

6. Battin, E.E. and Brumaghim, J.L. "Antioxidant activity of sulfur and selenium: a review of reactive oxygen species scavenging, glutathione peroxidase, and metalbinding antioxidant mechanisms", Cell Biochem. Biophys., 55(1), pp. 1-23 (2009).

7. El-Mously, M.K., Kotkata, M.F., and Salam, S.A. "Growth of crystalline phase in amorphous solid and liquid $\mathrm{SSe}_{20}$ ", J. Phys. C Solid State Phys., 11(6), pp. 1077-1083 (1978). 
8. Kotkata, M.F., Ayad, F.M., and El-Mously, M.K. "Photo-effect on crystallization kinetics of amorphous selenium doped with sulphur", J. Non-Cryst. Solids, 33(1), pp. 13-22 (1979).

9. Rai, M. and Posten, C., Green Biosynthesis of Nanoparticles: Mechanisms and Applications, CABI, UK (2013).

10. Dhanjal, S. and Cameotra, S.S. "Aerobic biogenesis of selenium nanospheres by Bacillus cereus isolated from coalmine soil", Microb Cell Fact., 9(1), pp. 1-11 (2010).

11. Ingole, A.R., Thakare, S.R., Khati, N.T., Wankhade, A.V., and Burghate, D.K. "Green synthesis of selenium nanoparticles under ambient condition", Chalcogenide Lett., 7(7), pp. 485-489 (2010).

12. Fesharaki, P.J., Nazari, P., Shakibaie, M., Rezaie, S., Banoee, M., Abdollahi, M., and Shahverdi, A.R. "Biosynthesis of selenium nanoparticles using Klebsiella pneumoniae and their recovery by a simple sterilization process", Braz. J. Microbiol., 41(2), pp. 461-466 (2010).

13. Li, S., Shen, Y., Xie, A., Yu, X., Zhang, X., Yang, L., and $\mathrm{Li}, \mathrm{C}$. "Rapid, room-temperature synthesis of amorphous selenium/protein composites using Capsicum annuum L extract", Nanotechnology, 18(40), pp. 1-9 (2007).

14. Zare, B., Babaie, S., Setayesh, N., and Shahverdi, A.R. "Isolation and characterization of a fungus for extracellular synthesis of small selenium nanoparticles", Nanomed. J., 1(1), pp. 13-19 (2013).

15. Sarkar, J., Dey, P., Saha, S., and Acharya, K. "Mycosynthesis of selenium nanoparticles", Micro Nano Lett., 6(8), pp. 599-602 (2011).

16. Abe, T., Hoshino, T., Nakamura, A. and Takaya, N. "Anaerobic elemental sulfur reduction by fungus Fusarium oxysporum", Biosci. Biotechnol. Biochem., 71(10) pp. 2402-2407 (2007).

17. Ahmad, A., Mukherjee, P., Mandal, D., Senapati, S., Khan, M.I., Kumar, R., and Sastry, M. "Enzyme mediated extracellular synthesis of CdS nanoparticles by the fungus, Fusarium oxysporum", J. Am. Chem. Soc., 124(41), pp. 12108-12109 (2002).

18. Mirzadeh, S., Darezereshki, E., Bakhtiari, F., Fazaelipoor, M.H. and Hosseini, M.R. "Characterization of zinc sulfide ( $\mathrm{ZnS}$ ) nanoparticles biosynthesized by Fusarium oxysporum", Master Sci. Semicond. Process, 16(2), pp. 374-378 (2012).

19. Pavia, D.L., Lampman, G.M., Kriz, G.S., and Vyvyan, J.A. "Introduction to spectroscopy", Cengage Learning, Boston, US. (2008).
20. Reyes, L.R., Gómez, I., and Garza, M.T. "Biosynthesis of cadmium sulfide nanoparticles by the fungi fusarium sp.", Int. J. Green Nanotech. Biomed, 1(1), pp. B90B95 (2009).

21. Wallenberg, M., Misra, S., and Björnstedt, M. "Selenium cytotoxicity in cancer", Basic Clin. Pharmacol. Toxicol., 114(5), pp. 377-386 (2014).

22. George, S., Infrared and Raman Characteristic Group Frequencies, John Wiley \& Sons Ltd., West Sussex, UK. (2001).

23. Rajput, S., Werezuk, R., Lange, R.M., and McDermott, M.T. "Fungal isolate optimized for biogenesis of silver nanoparticles with enhanced colloidal stability", Langmuir., 32(34), pp. 88-97 (2016).

24. Almeida, EdS, de Oliveira, Db, and Hotza, D. "Characterization of silver nanoparticles produced by biosynthesis mediated by Fusarium oxysporum under different processing conditions", Bioprocess Biosyst Eng., 40(9), pp. 1-13 (2017).

25. Yamaguchi, T., Tsuruda, Y., Furukawa, T., et al. "Synthesis of CdSe quantum dots using Fusarium oxysporum", Materials, 9(10), pp. 1-12 (2016).

26. Verschueren, K.H.G. and Wilkinson, A.J. "Sulfide: Biosynthesis from sulfate", ELS., 1(1), pp. 1-8 (2005).

27. Paietta, J.V. "Regulation of sulfur metabolism in mycelial fungi", Biochem. Mol. Biol., Springer, pp. 369-83 (2004).

\section{Biographies}

Farnoush Asghari-Paskiabi is a $\mathrm{PhD}$ candidate of Nanomedicine at Shahid Beheshti University of Medical Sciences, Tehran, Iran. She received her MSc in Nanomedicine from Tehran University of Medical Sciences in 2011. She has worked on nanofibrous scaffolds for skeletal muscle tissue engineering, antifungal nanofibrous wound dressings, and biosynthesis of selenium nanoparticles through fungi isolated from soils of sulfur-rich areas. She has carried out her $\mathrm{PhD}$ project on biosynthesis of selenium sulfide nanoparticles by fungal microorganisms under the supervision of Dr. Mohammad Imani, Prof. Mehdi Razzaghi-Abyaneh, and Prof. Hashem Rafii-Tabar.

Mohammad Imani is an Associate Professor of Pharmaceutics in Novel Drug Delivery Systems (NDDS) Department of Iran Polymer and Petrochemical Institute (IPPI). He was graduated in Pharmacy in 1996; then, he received a $\mathrm{PhD}$ degree in Pharmaceutics in 2002, prior to joining IPPI. His research interests include polymer synthesis and development, including new unsaturated aliphatic polyesters, furan resins, etc.; application of polymeric materials to develop controlledrelease drug delivery devices; and tissue engineering. Dr. Imani has engaged in some international research, focusing on development of novel drug-eluting cochlear 
implant devices, and is an advisor to some companies of drug-eluting biomedical devices. His research has been funded by government agencies and corporations. $\mathrm{He}$ is a co-inventor of three US patent applications.

Mehdi Razzaghi-Abyaneh obtained his PhD degree in Mycology from Tarbiat Modares University, Iran, in 2000. He pursued a training course on identification of antifungal compounds from bioactive plants in the Laboratory of Applied Biological Chemistry at the Graduate School of Agriculture, Tokyo University, during 2006-2007. He is currently a full professor and head of Mycology Department at the Pasteur Institute of Iran (Tehran, Iran), where he has been working on mycotoxins and mycotoxigenic fungi for more than 15 years. His research interests encompass biologically active compounds of plant, fungal, and bacterial origins with antifungal properties against a broad spectrum of fungal pathogens. He has supervised a large number of $\mathrm{PhD}$ and $\mathrm{MSc}$ theses. He has published over onehundred papers in peer-reviewed international journals, eight books, and a number of book chapters.

Hashem Rafii-Tabar is a Professor of Computational Nano-Science and Nano-Technology in the Department of Medical Physics and Biomedical Engineering, Faculty of Medicine, Shahid Beheshti University of
Medical Sciences. He was the founder and head of Nano-Technology Committee, Ministry of Science, Research and Technology, Iran, during 2001-2002; head of Research in Computational Nano-Science Research Section, Centre for Numerical Modelling and Process Analysis, University of Greenwich, England, during 1994-2000; and invited Research Professor in Computational Nano-Science, Institute for Materials Research, Tohoku University, Japan, during 1992-1993. He was senior research fellow in Computational Nanoscience in the Department of Materials, University of Oxford, 1990-1992. He was an Associate Professor in Mathematics and Computer Science, South West London College, during 1989-1992, and was a visiting Research Physicist in Foundation of Quantum Mechanics, Henri Poincari Institute, Paris, during 1984-1987, and in United Nations (UN) Research and Educational Consultant, during 1994-1995. He was a co-chair of the Physics and Economic Development Committee, World Conference on Physics and Sustainable Development, South Africa, in 2005, and Head of the International Network on Nanotechnology Projects, supported by UN. He has published more than 100 research papers in five fields of theoretical physics and nano-science, and is the author of the first book entitled "Computational Physics of Carbon Naotubes" published by Cambridge University Press in 2008. 